\title{
Fuzzy traffic light controller using Sugeno method for isolated intersection
}

\begin{abstract}
This paper presents a Sugeno-type fuzzy traffic controller for controlling isolated traffic intersection. The model framework used in this study developed using $M / M / 1$ queuing theory. The principles and rules for the fuzzy control system are modeled based on the arrivals of vehicles, vehicles queue length at current green phase, and vehicles queue lengths at the other phases. The phase sequence and phase length extension are used in the decision making to switch the current phase to the appropriate next phase. Using the information from its traffic detectors at each of the four lanes of isolated intersection, the fuzzy rule-based system gives optimal signals to adapt the phase lengths to the traffic conditions. The phase sequence is managed by a simple program using if-else statement. In this paper, an isolated traffic intersection is simulated for both fixed-cycle traffic control system and fuzzy logic based traffic control system. The comparison of performance between the two models of traffic light system clearly indicates that the fuzzy logic based traffic lights control system is more efficient, flexible and adaptable in controlling the traffic flow.
\end{abstract}

Keyword: Fuzzy traffic controller; Phase length extension; Phase sequence; Sugeno-type 